\title{
Techniques, Technology and Tekne: The Ethical Use of Guidelines in the Practice of Interventional Pain Management
}

\author{
James Giordano, PhD
}

Guide: v, transitive: (from Old French guider - to direct; from Teutonic, and akin to Old English wise); to lead or direct any course of action or practice (as by advice, wishes or prudence) (1).

$\square$ his issue of Pain Physician is dedicated to the dissemination of reviews and guidelines that are evidencebased, and that seek to provide, by definition, some level of direction in the specific actions of interventional pain practice. The work upon which these guidelines are structured focused upon the pathologic bases of particular pain disorders and syndromes, the techniques and technologies that constitute specific therapeutic interventions, and the outcomes that such interventions produce in representative populations. In many ways, this information affords theoretical knowledge (of various pain pathologies and techniques of diagnosis and intervention), empirical information (regarding the outcomes of these interventions), and contextual focus (by grounding outcomes' evidence to particular patient-characteristics and specifics). This provides a framework for the decisional processes that shape the clinical practice of pain medicine.

I argue that guidelines (and the decisional processes they facilitate) must be built upon a progressively revised understanding of pain, recognition and appreciation of its effects, and acknowledgment of the ca-

Deputy-Editor-in-Chief Pain Physician

Scholar in Residence, Center for Clinical Bioethics; Associate

Professor, Department of Palliative Medicine, Georgetown University Medical Center, Washington, D.C.

Author correspondence: James Giordano, PhD, Center

for Clinical Bioethics, 4000

Reservoir Rd., Bldg D,

Washington, DC 20057

E-mail:

jgiordano@neurobioethics.org

Support: This work was

supported, in part, by the

Center for Clinical Bioethics

and Department of Palliative

Medicine, Georgetown

University Medical Center, and the Manchikanti Distinguished

Lectureship of the American

Society of Interventional Pain Physicians.

Free Full manuscript: www.painphysicianjournal.com pacities and limitations of contemporary medicine.

From a practical standpoint, such clinical guidelines could provide a sustainable set of directions that can be employed in a decision-making process. Given the assumption that there is constant change (in knowledge, parameters of practice, and the economic, political, and social milieu in which practice occurs), sustainability is determined on three levels, epistemic (ie, knowledge-based), experiential, and applied (2). The epistemic component is maintained, at least in part, by 1 ) ongoing research and assessment of new information, 2) continuing incorporation of such information, so as to allow 3) constant revision of the guidelines themselves to reflect the most contemporary findings and knowledge. This implies that guidelines must be part of a progressive learning process, in which new information supersedes old, and relative values (of particular techniques, technologies, procedures etc.) are re-appraised, re-affirmed, and/or revised to be resonant with those of the participant communities. From this it can be seen that for guidelines to be sustainable, they must be dynamic and part of a paradigm of change (3). But recall that for change to be effective, it must be preceded by learning. In the Hippocratic tradition, the perdurablity of learning, as a process, involves incorporation of knowledge of the natural phenomenon (ie, pain), and bring to this new information using experience and wisdom $(4,5)$. As described by Mark Meaney (6), the incorporation of the four phases of experiential learning (eg. "...description, design, development, and doing") (7) into a larger decisional process model that accounts for principles, participants, potential, practice(s), and ongoing review has been elucidated by Brown (8), and possesses significant merit for use within healthcare. This expanded model, known as D4P4, not only reflects 
much of the mechanics of guideline development (ie, the "D4" process), but also affords mechanisms that allow accountability, revision, and support ethical use (ie, the "P4 and review" process). As Meaney notes, the D4P4 process has been successful in stimulating research, development of improved models, tools and methods, promotion of safe use of new techniques and technologies, and engagement of public involvement and risk management policy (6).

The development, incorporation, and revision of interventional pain management guidelines fit well within a D4P4 model, in light of the inherent difficulties of pain management, as a practice. Recently we examined the problematic nature of practical pain management, illustrating the contributory role(s) played by the complicated pathology and often enigmatic nature and expression of pain, economic factors, and the current medico-legal environment (9). The guidelines provided in this volume can certainly assist in navigating this terrain. But clinical guidelines alone, while necessary, are not sufficient to account for the complexity of the encounter between the physician and the patient in pain, and the decisional processes and responsibilities borne by the physician within this relationship. To be sure, guidelines must account for, and often counter-balance, economic influences, and what Ziman calls "the norm of utility" that involves both cost-effectiveness incentives and human values and concerns (10). This is increasingly important in light of the availability (and incentives for use) of newer, more advanced technologies, and technologically based procedures, the tendency toward a somewhat technocentric valueladeness, and mounting pressure(s) of an increasingly pervasive market model mentality of much of modern medicine (11-13). I most assuredly do not advocate a Luddite divorce from any or all things technological. Quite the contrary, as technology has provided modern medicine with the tools to make remarkable progress in diagnosis and treatment. But, as Reiser notes "...technologies...can be imperative: we may be impelled to use the capacities they provide us without adequate reflection on whether they will lead to the humane goals of medical care" (14). Questions and guidance on why, how, when, and what technology and technologically based technique(s) should be utilized are often more important than simply whether or not these can be used. In dealing with technological developments, Paul Cilliers states that "...powerful technology is not an unconditional blessing," rather, while affording certain ease, it demands that we become more stringent in understanding its use, limitations, and de-limitations in specific, rather than purely generalized contexts (15). Notes Cilliers: "...that demands new ways of thinking...in this sense philosophy has an important role to play...by being an integral part of scientific and technological practice" (15).

It is in this context that guidelines must be informed by a (neuro)philosophy of pain, and must equally and reciprocally reflect and sustain the philosophical basis of medicine, in general, and pain medicine more specifically (16). Any philosophy incorporates epistemic, anthropologic, and ethical domains, and I hold that the philosophy of pain, and pain medicine is no different $(17,18)$. Contemporary understanding of pain informs each of these domains: by depicting pain and suffering as complex, fundamental harms that are experienced in often uniquely individual ways by autonomous, sentient persons, who are the moral focus of the clinical encounter, this establishes the realities, the premises, ends and claims of pain medicine as a practice-an exchange of "good" between moral individuals that reflects the intent and nature of their relationship(19). The facts of pain and realities of pain medicine also contribute to what I believe to be the duties and obligations (ie, the deontic foundation) inherent to the "core structure" of pain medicine as a practice on both an individual and public level $(20,21)$. These facts and realities define this practice pragmatically and morally, and must be used to inform both clinical guidelines and policies that serve individuals and society (Fig. 1).

Pragmatically, the practice 1) is axiomatically focused upon relieving and effectively managing pain; 2) involves the use of extant and developing therapeutic techniques and technologies in ways that are resonant to our current epistemic appreciation of pain (as symptom, disease, and illness), and at the same time responsive to the clinical needs of individual patients. Morally, the "good" of this practice reflects the fact that medicine, in general, involves humanitarian actions provided by the physician (as therapeutic and moral agent) to the person in pain (as a vulnerable moral patient and subject of responsibility), and within a larger realm, sustains the role of medicine as a public good (20-22). More specifically, the moral obligations of pain medicine entail 1) the fiduciary that is based upon the physician's professed intentions and abilities to relieve pain and suffering; 2 ) an appreciation that each person's pain is (in many ways) unique, subserved by changes in the neuraxis that affect sensation, perception, cognition, emotion, and behavior; and 3) the primacy of each pain patient's best interests in deter- 


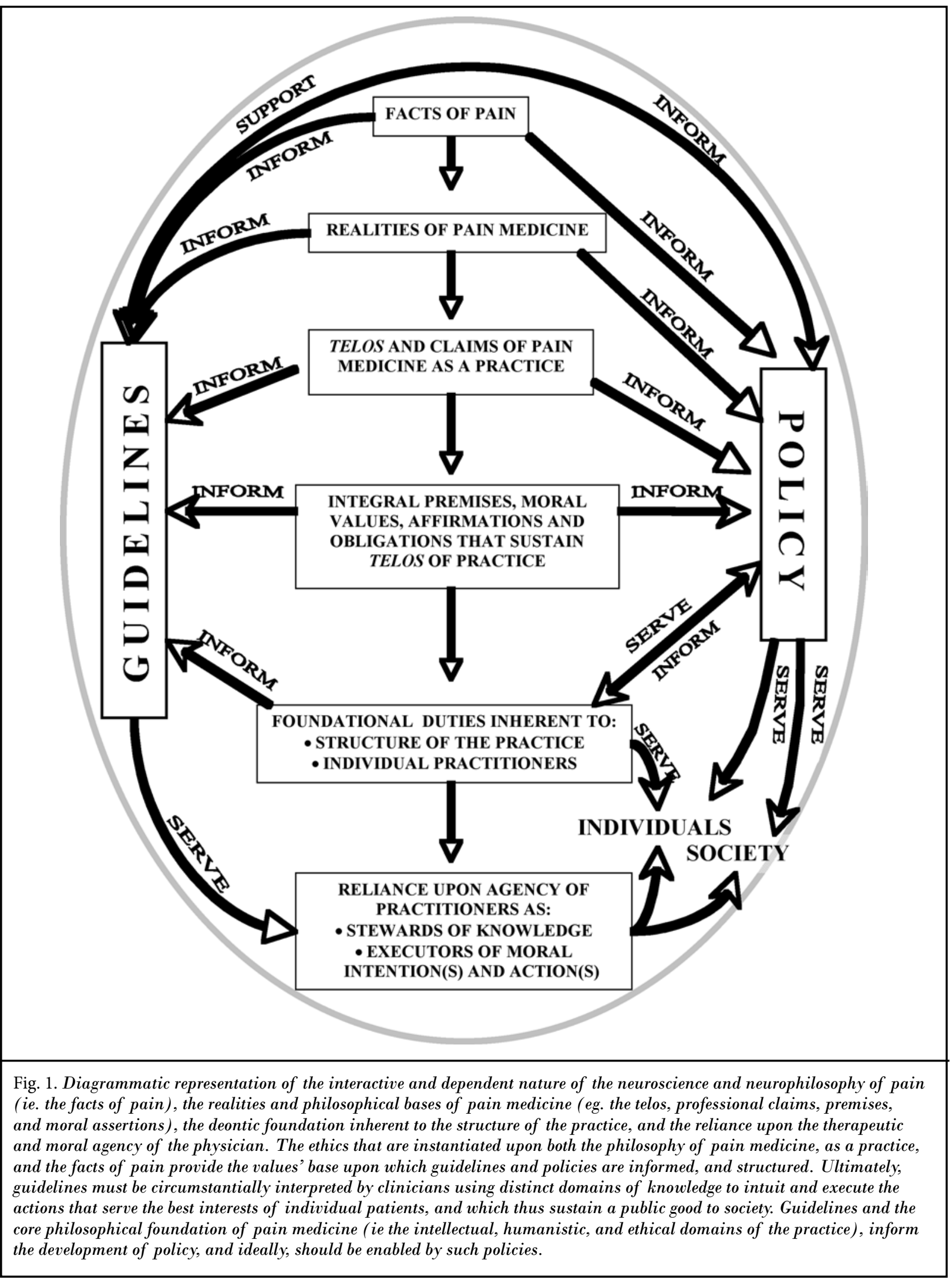


mining the type, extent, and scope of care that is rendered (18).

While a deontic foundation, that is, a set of rules, can sustain the structure of what pain medicine is supposed to "do," the actual intimate interaction between a specific physician and patient cannot be wholly maintained by categorical rules or principles. In fact, I have posited that one of the cardinal "rules" of pain medicine is that primary accord must be given to the relational nature of the physician as moral agent and the person in pain as moral patient $(20,21)$. In other words, the "rules" of the practice of pain medicine "state" and fortify the importance and essential value of the physician's individual moral agency. Any interaction is a calculus of circumstance, moral agents (and patients), intentions, actions, and consequences (23). Therefore, it can be seen that even the most rigorous guidelines must account for the ability of the physician (as steward of diverse types and depths of knowledge) to intuit the intricacies of information, values, and circumstance with wisdom that is grounded in the best interest(s) of the patient (24), and reflective of the ends of pain medicine as a curative, healing, and/or caring practice. Indeed, the guidelines provided in this issue provide a basis for the use of new information, and can assist in directing the utility of key skills. But medicine is not simply applied skill; it is skill enabled by art (ie, a tekne) (25). The "art" of medicine, as a rational discipline in the Hippocratic tradition, is what differentiates the practice from other, purely theoretical and/or mechanical enterprises $(25,26)$. The utility of guidelines is that they provide a template that afford insight into what can be done, given generalized clinical situations. Ultimately, what should be done must be ascertained by the physician by evaluating and weighing the circumstantial, personal, clinical, and often socio-legal variables that constitute decisional process within the clinical encounter.

For at the root of even the most pragmatic applications of the decisional process is the need to describe the circumstances fully, orient the moral compass with respect to professional values and those of the participant persons (eg. patients, families, et al.), consider potential outcomes, and engage in the process of resolving equipoise. Thus, it can be seen that the sustainability of guideline use within the D4P4 model establishes a primary role for ethical consideration that is based upon the philosophical premises and defined ends and claims of pain medicine. Guidelines can serve as a roadmap for clinical care, but the
Table 1. Sequential questions and steps in the methodological approach to resolution of ethical dilemmas.

1. What are the facts?

-diagnosis

-prognosis

-therapeutic options available

-biography of the patient

-chronology of event

-interests/issues of other involved parties

-clinical settings

2. What are the issues?

-are the issues truly "ethical"?

-are the issues due to communication problems?

-are the issues clinical issues, narrowly defined?

3. How should the issues be framed?

-in terms of the good of the patient

i. patients' ultimate good

ii. patients' good as a person

iii. good of the patients' choices

iv. biomedical good of/for the patient

-in terms of good and interests of other involved parties

i.family

ii. community, society and the law

iii. healthcare institution(s)

iv. members of the conjoined medical team

4. How are the issues positioned relative to comparable cases?

5. What can and should be done?

-evaluate options and ethically relevant considerations

i. balance of benefits and harms

ii. "cost" effectiveness and allocation

iii. issues of cultural and religious variation

iv. considerations of power structures/relationships

-grounding of options and source(s) of ethics

6. What are the merits and drawbacks of this possible solution?

-evaluation of viable options for resolution

-establishment of decision-basis or consensus

7. Decide upon a right and good resolution.

-post-facto critique and analysis

legend that enables using such a map is moral. Irrespective of the ethical system(s) used, I have argued that the intellectual virtue of practical wisdom (ie, phronesis) is essential to determine the right knowledge to empower the right decisions that enable the right actions in any given circumstance $(18,20,21,27)$. Each step in the decisional algorithm must be weighed not only for its clinical benefit (ie, the biomedical good), 
but for the effect(s) that this decision will incur upon the personal good of the patient, in light of his/her choices, circumstance(s), and values (24). In sum, each step along any guided path involves ethical decisionmaking. Surely, there will be situations in which values differ, and a variety of ethical approaches will be necessary to facilitate the decisional process. To some extent, guidelines for ethical decision-making can be used to enable this, and the 7-step process for ethical evaluation provided in Table 1 can be valuable toward these goals. The philosophical premises and ethical evaluations that are instantiated thereupon are not "after-the-fact" events to the clinical decision-making process, but are intrinsic parts of the process itself, establishing the values and moral affirmations that lead this procedure.

But even for such ethical guidelines to be accurately and effectively employed, they must be interpreted by individuals who are not merely automatons of protocol, but who use practical wisdom to exercise the moral responsibilities of determining and provid- ing the "good" of the practice. To be sure, the process of guideline use and ethically sound, clinically prudent decision-making is arduous and demanding. The development of clinical (and ethical) guidelines necessitates progressive research, ongoing revision, and the right and good use of any such guidelines must be instilled through education and training. Yet, as demanding as these assurances may be, they reflect the responsibilities of the pain physician to utilize new information, expand professional knowledge to be consistent with a progressively changing epistemology, and adhere and respond to the moral obligations and affirmations of good and non harm (28). As Reiser reminds us, this issue was paramount to the Hippocratic tradition (14), and like Reiser, I believe that it is no less meaningful to the practice of good medicine today.

\section{Acknowledgments.}

The author would like to thank Sherry Loveless for graphic artistry.

\section{References}

1. The New Century Dictionary, vol 1, Emery HG, Brewster KG (eds.) D. AppletonCentury Co., NY 1940 p. 694.

2. Gibbons $M$, Limoges $C$, Nowotny $\mathrm{H}$, Schwartzman S, Scott P, Trow M. The Production of New Knowledge. Sage, London, 1994.

3. Institute of Medicine. Who Will Keep the Public Health? Educating Public Health Professionals for the 21st Cen tury. National Academies Press, Washington, DC, 2003.

4. Edelstein L. Ancient Medicine: Selected Papers. Temkin 0 , Temkin CL (eds.) Williams and Wilkins, Baltimore 1967.

5. Smith WD. The Hippocratic Tradition. Cornell University Press, Ithaca, 1979.

6. Meaney ME. Lessons from the sustainability movement: Toward an integrative decision-making framework for nanotechnology. I Law, Medicine and Ethics, 2006; 34(4):682-688.

7. Kolb D, Rubin I, Mclntyre J. Organizational Psychology: An Experiential Approach. Prentice-Hall, NJ, 1974.

8. Brown V. (ed.) Managing for Local Sustainability: Policy Problem-solving, Practice and Place. University of Western Sydney Press, Richmond, Australia, 1997.

9. Giordano J. LeRoy P, Uthaman U. On the role of primary care within a system of integrative multi-disciplinary pain management. Prac Pain Management 2006, 6(8): 66-69.

10. Ziman J. Post-academic science: Constructing knowledge with networks and norms. Science Studies, 1996; 9: 67-80.

11. Tiles M, Oberdiek H. Living in a Technological Culture: Human Tools and Human Values. Routledge, NY, 1995.

12. Herzlinger RE. Market-driven Healthcare. Perseus Books, Reading MA, 1997.

13. Giordano J. Cassandra's Curse: interventional pain management, policy and preserving meaning against a market mentality. Pain Physician, 2006; 9: 167-170.

14. Reiser SJ. Technology. In: Post SG (ed.) Encyclopedia of Bioethics, 3rd Ed., vol. 5, Thomson-Gale, NY, 2004, p. 2502.

15. Cilliers P. Complexity and Postmodernism. Routledge, NY, 1998. p. 1-2.

16. Giordano J. Understanding pain as disease and illness-Part One. Prac Pain Management, 2006; 6(6): 70-73.

17. Maclntyre A. The Tasks of Philosophy. Vol. 1, Cambridge University Press, Cambridge, 2006.

18. Giordano J. Moral agency in pain medicine: philosophy, practice and virtue. Pain Physician, 2006; 9: 41-46.

19. MacIntyre, A. After Virtue. 2nd Ed. University of Notre Dame Press, Notre Dame, IN, 1984.
20. Maricich Y, Giordano J. Pain suffering and the ethics of pain medicine: Is a deontic foundation sufficient? Am J Pain Management, 2006; 16: 130-138.

21. Giordano J. Pain as disease and illness Part Two. Prac Pain Management, 2006; 6(7): 65-68.

22. May WF. The Physician's Covenant. Westminster Press, Philadelphia, 2000.

23. Aristotle. Nicomachean Ethics. T. Irwin (trans) Hackett Publishing, Indianapolis, 1996.

24. Pellegrino ED, Thomasma DC. For the Patient's Good: The Restoration of Beneficence to Health Care. Oxford, NY, 1988.

25. Hippocrates. The Art. Hippocrates II. WHW Jones (trans.) Loeb Classical Library 148, Harvard University Press, Cambridge MA, 1981.

26. Aristotle. Posterior Analytics. T. Irwin, G. Fine (trans.) Hackett Publishing, Indianapolis, 1996.

27. Giordano J. Pain, the patient, and the practice of pain medicine. The importance of a core philosophy and virtue based ethics. In: Schatman M (ed.) Ethics in Chronic Pain Management. Informa, NY, 2006, p. 1-18.

28. Giordano J. Ethical obligations in infrared imaging research and practice. In: Diakedes N (ed.) Functional Infrared Imaging. Informa, NY, in press. 
